\title{
HIGH ACCURACY INTERFACE FOR SENSOR BRIDGES
}

\author{
C. FALCONI, A. D’AMICO, E. MARTINELLI, G. SAGGIO, C. DI NATALE \\ Department of Electronic Engineering, University of Tor Vergata, \\ Rome, Italy
}

\begin{abstract}
The ideal electronic interface for sensor bridges should introduce small offset and noise and, possibly, be linear with respect to the relative resistance change. Here we introduce a new method which, in comparison with previously reported techniques, is simpler, has better sensitivity and allows a better rejection of non linearity. Furthermore the proposed approach makes it easy the compensation of component mismatch by dynamic element matching.
\end{abstract}

\section{Introduction}

For a resistive sensor we may consider

$$
R=R_{0}+\Delta R=R_{0}(1+\delta)
$$

An electronic interface for a resistive sensor should produce a voltage signal related to the relative resistance variation, $\delta$. In some applications a non-linear dependence of the output voltage on the relative resistance variation is allowed because, for instance, a digital microprocessor may easily take such non linearity into account. However a good linearity of the sensor response is often a fundamental requirement (for instance in many smart sensors systems).

Electronic interfaces for resistive sensors often use resistive bridges. Figure 1 shows a simple resistance bridge which produces an output voltage depending on $\delta$ in an exactly linear fashion [1,2]; however, since in many cases sensors whose impedance variations are opposite are not available, the circuit shown in figure 1 is not always practical.

Recently, an alternative approach (see figure 2), based on a floating negative impedance converter has been proposed [3]; a residual non-linearity is present as shown in figure 3 (non-linearity error vs. the resistance variation, being $R_{0}=10 \mathrm{k} \Omega, V_{R E F}=5 \mathrm{~V}$ ). Beside this residual non-linearity, the practical implementation of this solution is not straightforward (a fully differential op amp is required) for systems using discrete components and expensive (chip area) for integrated systems. 


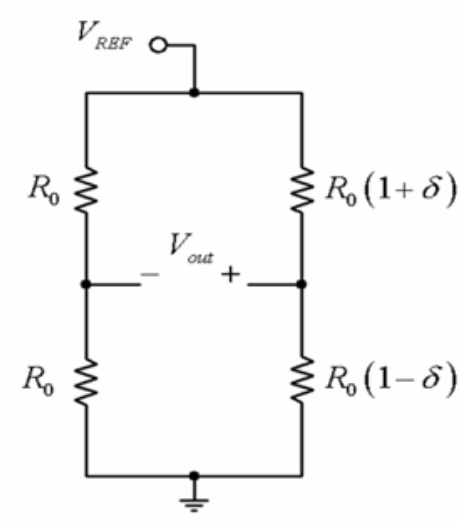

Fig. 1 Electronic interface for resistive sensors.

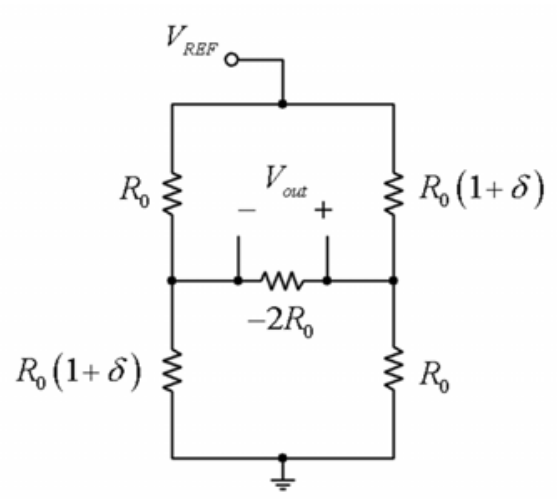

Fig. 2 Electronic interface for resistive sensors proposed in [3].

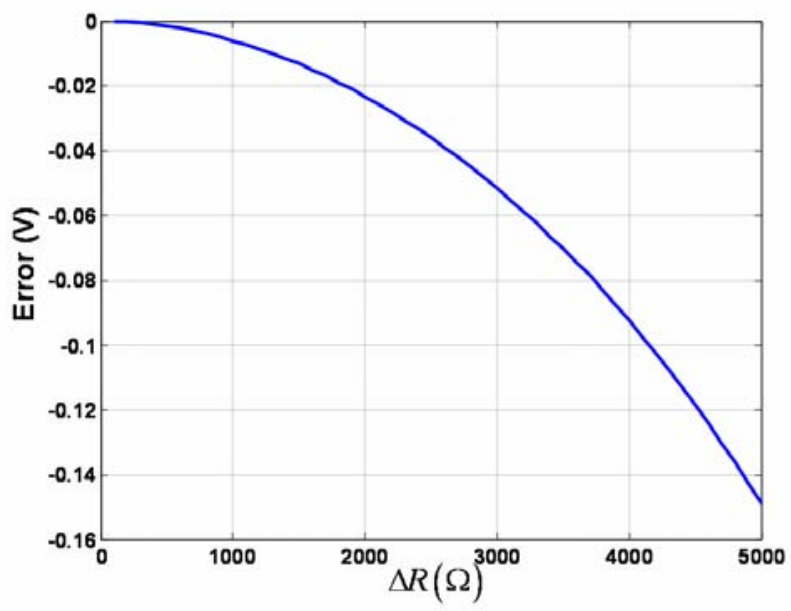

Fig. 3 Non linearity error vs. the resistance variation in the circuit shown in fig. 2 


\section{High Accuracy Interface for Sensor Bridges}

Figure 4 shows a circuit, based on a grounded negative impedance converter, whose output voltage depends on $\delta$ in an exactly linear fashion (this circuit, in fact, is equivalent to the ideal circuit shown in figure 1); the sensitivity of this interface is doubled when compared with the circuit [3] and the implementation of a grounded (voltage mode) negative impedance converter only requires a standard op amp (see figure 5). Figure 6 shows the non-linearity error when a low cost TL082 op amp is used.

In integrated sensor systems, the technique here reported may be easily combined with dynamic op amp matching (figure 7 shows the input stage of an instrumentation amplifier based on this approach [4,5]) and with dynamic element matching applied to the resistive sensors, in order to reject mismatch of both the sensors and the op amps. The proposed approach is not limited to resistive sensors and may be applied, with proper modifications, to other types of impedance sensors.

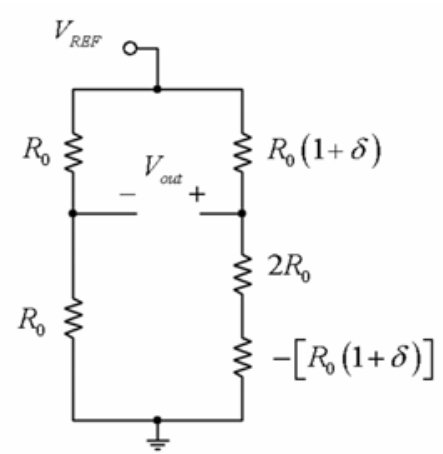

Fig. 4 Proposed solution for implementing the circuit shown in fig. 1

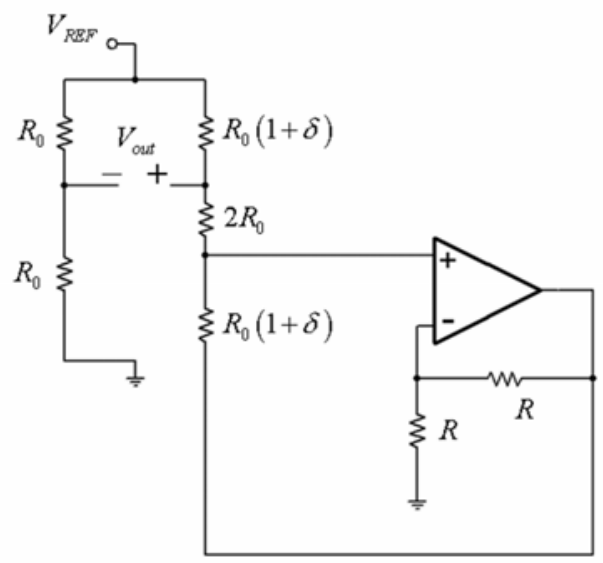

Fig. 5 Practical implementation of the proposed solution shown in fig. 4. 


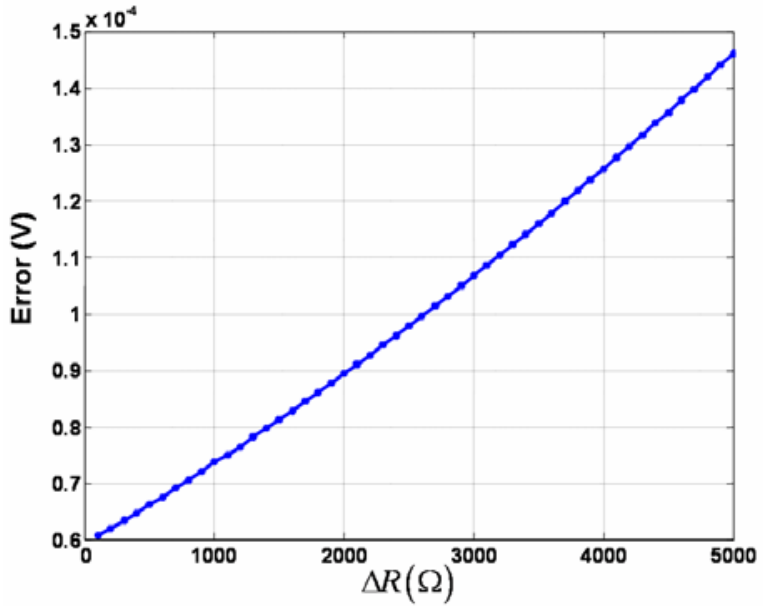

Fig. 6 Non linearity error vs. the resistance variation in the circuit shown in fig. 5 using a low cost TL082 op amp.

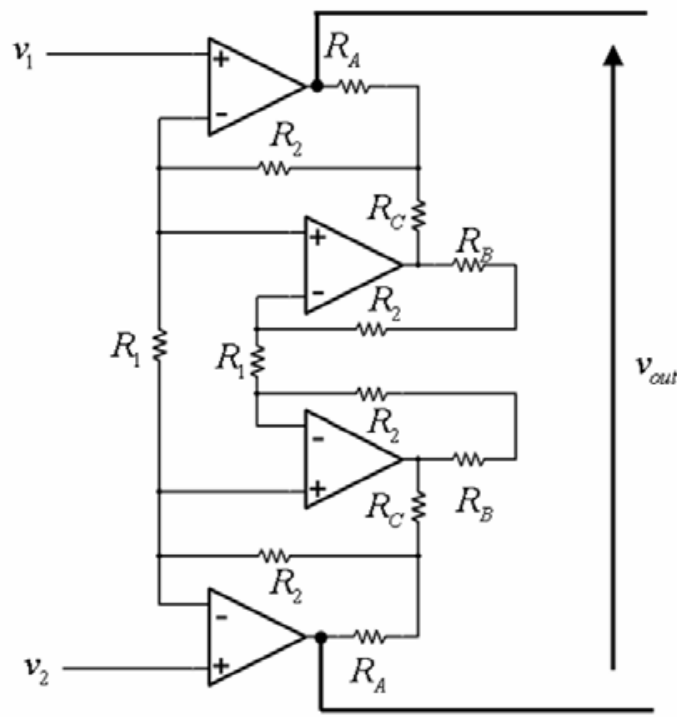

Fig. 7 The input stage of an instrumentation amplifier with compensation (after dynamic op amp matching) of the input offset and $1 / \mathrm{f}$ noise voltages and of the finite op amp gain. 


\section{Conclusions}

Here we have proposed a new circuit which takes advantage of a grounded negative impedance converter for producing an output voltage which depends on the relative impedance variation of an impedance sensor in an exactly linear fashion (neglecting the non idealities of circuit components). The technique may be applied for both discrete and integrated systems; in the latter case, it can be easily combined with dynamic element matching and/or dynamic op amp matching.

\section{References}

1. R. Pallàs-Areny, J. G. Webster, "Sensors and Signal Conditioning”, Wiley, 2001

2. S Middelhoek, S. A. Audet, "Silicon Sensors”, Academic Press Limited,London,1989

3. G. de Graaf, R. F. Wolffenbuttel, "New concept for the linearisation of sensor bridge circuits", Proceedings of the XVII Eurosensors Conference, Lisbona (Portugal), 2003

4. C. Falconi, A. D'Amico, M. Faccio, "Design of accurate analog circuits for low voltage low power CMOS systems", Proceedings of IEEE ISCAS 2003, vol. 1, pp. 429-432.

5. C. Falconi, M. Faccio, A. D’Amico, C. Di Natale, "High accuracy instrumentation amplifier for low voltage low power CMOS smart sensors”, Proveedings of ISCAS 2003, Bangkok, Thailand

6. C. Falconi, C. Di Natale, A. D’Amico, M. Faccio, "Electronic interface for the accurate read-out of resistive sensors in low voltage-low power integrated systems”, in press, Sensors and Actuators, 2004

7. C. Falconi, "High accuracy electronic interfaces for CMOS Microsystems", Proceedings of Eurosensors 2004, Rome, Italy 